\title{
Decreased SUMOylation of the retinoblastoma protein in keratinocytes during the pathogenesis of vitiligo
}

\author{
MIAONI ZHOU, FUQUAN LIN, WEN XU, RONG JIN and AIE XU \\ Department of Dermatology, Hangzhou Institute of Dermatology and Venereology, \\ Third People's Hospital of Hangzhou, Hangzhou, Zhejiang 310009, P.R. China
}

Received November 17, 2017; Accepted March 12, 2018

DOI: $10.3892 / \mathrm{mmr} .2018 .9299$

\begin{abstract}
The role of SUMOylation in the pathogenesis of vitiligo has not been reported previously. The present study aimed to reveal abnormalities in small ubiquitin-like modifier (SUMO) conjugation in keratinocytes from depigmented lesions of patients with vitiligo and confirm the role of SUMOylation in keratinocytes from patients with vitiligo. Skin samples used for immunohistochemistry were obtained by punch biopsy from the depigmented lesions of 6 patients. Blisters were produced by vacuum and the roofs were collected for keratinocyte culture. HaCaT cells were transduced with SUMO1 knockdown vectors. The protein expression of SUMO1, SUMO-specific peptidase 1 (SENP1), ubiquitin-conjugating enzyme E2 I (Ubc9), SUMO-activating enzyme subunit 1 (SAE1), cyclin-dependent kinase (CDK)2, CDK6, proliferating cell nuclear antigen (PCNA), retinoblastoma protein $(\mathrm{Rb})$, phosphorylated $\mathrm{Rb}(\mathrm{pRb})$ and $\beta$-actin was assessed by western blotting. The SUMOylation status of proteins was assessed by immunoprecipitation. Cell cycle analysis was performed by flow cytometry and cell proliferation rate was investigated using a Cell Counting Kit-8. The results demonstrated that the levels of SUMO1-conjugated proteins were decreased in vitiligo lesions and vitiligo keratinocytes compared with normal controls. The protein expression of Ubc9 was decreased and SENP1 was increased in vitiligo keratinocytes compared with normal keratinocytes, with no alterations in SAE1 expression. Following knockdown of SUMO1 in HaCaT cells, the proliferation of HaCaT cells was reduced and the cell cycle was arrested in G1 phase.
\end{abstract}

Correspondence to: $\mathrm{Dr}$ Aie Xu, Department of Dermatology, Hangzhou Institute of Dermatology and Venereology, Third People's Hospital of Hangzhou, 38 Xihu Road, Hangzhou, Zhejiang 310009, P.R. China

E-mail: xuaiehz@msn.com

Abbreviations: CDK, cyclin-dependent kinase; SCF, stem cell factor; IHC, immunohistochemistry; pRb, phosphorylated retinoblastoma; SUMO, small ubiquitin-like modifier

Key words: vitiligo, melanocytes, keratinocytes, small ubiquitin-like modifier 1, retinoblastoma protein
Furthermore, the protein expression levels of PCNA, CDK2, CDK6 and pRb were reduced in SUMO1-knockdown HaCaT cells, and SUMOylated $\mathrm{Rb}$ was also decreased markedly in keratinocytes from lesions of patients with vitiligo compared with normal keratinocytes. In conclusion, vitiligo lesions in the present study exhibited dysregulated SUMOylation and deSUMOylation balance and dysregulation of cell cycle progression may be present in SUMO1 knockdown HaCaT cells. These results indicate that deSUMOylation of $\mathrm{Rb}$ of keratinocytes may serve an important role in vitiligo, providing a novel direction for the study into the mechanism of vitiligo.

\section{Introduction}

Vitiligo is a chronic, acquired pigment skin disorder, clinically characterized by well-defined asymptomatic white macules resulting from a loss of functional melanocytes in the epidermis (1). The majority of previous studies on vitiligo have focused on melanocyte abnormalities, but the epidermis structural unit consists of melanocytes with neighboring keratinocytes, and direct cell-to-cell contact and growth factors produced by adjacent keratinocytes regulate the differentiation, proliferation and cell viability of melanocytes (2). Keratinocytes secrete numerous factors that are essential to the survival of melanocytes, which include basic fibroblast growth factor, endothelins, stem cell factor (SCF), hepatocyte growth factor, granulocyte-macrophage colony-stimulating factor, $\alpha$-melanocyte-stimulating hormone and adrenocorticotropic hormone (3). Previous studies have demonstrated that co-culture of normal human melanocytes with human keratinocytes leads to an increased number of melanocytes $(3,4)$. Therefore, keratinocytes serve an important role in the homeostasis of the epidermis and survival of melanocytes.

Previous studies have revealed that the keratinocytes in depigmented regions of the epidermis in patients with vitiligo exhibited ultrastructural and functional alterations of their mitochondria (5) and had high apoptotic rates (6). In addition, significantly lower expression of SCF has been reported in apoptotic keratinocytes induced by ultraviolet radiation or $\mathrm{H}_{2} \mathrm{O}_{2}$ treatment (6). Keratinocyte apoptosis may lead to a loss of survival stimuli and passive melanocyte death. Therefore, it is important to investigate the specific mechanisms of keratinocyte apoptosis in patients with vitiligo and to identify methods to improve the biological activity of keratinocytes 
in order to alter the local microenvironment of depigmented lesions and enhance the vitality of the local melanocytes.

Among the proteins involved in the cellular biological activity of mammalian cells, including keratinocytes, the small ubiquitin-like modifier (SUMO) is a conserved member of the ubiquitin-related protein family and activates various cellular events, including protein trafficking, cell cycle and chromosome structure and segregation, by conjugating to numerous protein substrates (7-9). SUMOylation and deSUMOylation are dynamic processes. SUMOylation is controlled by an E1-activating enzyme [SUMO-activating enzyme subunit (SAE)1/2], an E2 conjugase [ubiquitin-conjugating enzyme E2 I (Ubc9)] and, in certain cases, the E3 ligases, while deSUMOylation is mediated by SUMO-specific peptidases (SENPs) (10). Previous studies have indicated that the SUMO pathway may be essential for cancer cell survival and tumor progression (11-13). Therefore, SUMOylation may be an important regulator for keratinocyte differentiation and survival, and the modulation of melanocytes (14).

To the best of our knowledge, the role of SUMOylation in the pathogenesis of vitiligo has not been reported previously. The current study hypothesized that abnormal apoptosis of keratinocytes in patients with vitiligo may be associated with the SUMO modification status of these cells. The present study aimed to reveal abnormalities in SUMO conjugation in keratinocytes from depigmented lesions of patients with vitiligo and confirm the role of SUMOylation in keratinocytes from patients with vitiligo.

\section{Materials and methods}

Tissue samples. Skin samples used for immunohistochemistry (IHC) were obtained by punch biopsy under local lidocaine anesthesia from depigmented lesions of vitiligo patients ( $n=6 ; 2$ male and 4 females) aged 16-37 years. All vitiligo patients had been diagnosed by dermatologists and reflectance confocal microscopy, according to published criteria $(15,16)$ : Hypopigmentation lesion of the skin with no associated inflammation; wood lamp examination accentuating the vitiligo lesion; biopsy showing complete absence of melanocytes with loss of epidermal pigmentation. Matched normal adult human skin specimens were obtained from 6 healthy adults ( 2 male and 4 female) aged 18-41 years undergoing plastic surgery.

Blisters ( $8 \mathrm{~mm}$ in diameter) were produced with a vacuum of $40 \mathrm{kPa}$ for $60-90 \mathrm{~min}$ in healthy individuals and vitiligo patients (from depigmented areas). Blister roofs were collected and used for the isolation and culture of keratinocytes and protein analysis.

The present study was approved by the Institutional Review Board of the Third People's Hospital of Hangzhou (Hangzhou, China). Informed consent was obtained from each participant.

$I H C$. Skin tissues were fixed in $4 \%$ paraformaldehyde at room temperature for $20 \mathrm{~min}$. IHC analysis was performed on $4-\mu \mathrm{m}$ thick sections from paraffin-embedded clinical samples. Tissue slides were preheated in a $60^{\circ} \mathrm{C}$ oven for $2 \mathrm{~h}$. The sections were immersed in xylene (I) at room temperature for $30 \mathrm{~min}$, followed by xylene (II) to deparaffinize at room temperature for $10 \mathrm{~min}$. Slides were subsequently immersed into $100 \%$ ethanol for $10 \mathrm{~min}$, $95 \%$ ethanol for
$5 \mathrm{~min}, 90 \%$ ethanol for $2 \mathrm{~min}, 80 \%$ ethanol for $2 \mathrm{~min}, 70 \%$ ethanol for $2 \mathrm{~min}$ and double distilled water for $2 \mathrm{~min}$, all at room temperature. Slides were rinsed in PBS-T (0.01M PBS, $\mathrm{pH} 7.4 ; 0.02 \% \mathrm{KH}_{2} \mathrm{PO}_{4}, 0.29 \% \mathrm{~N}_{2} \mathrm{HPO}_{4}, 0.02 \% \mathrm{KCl}, 0.8 \%$ $\mathrm{NaCl}, 0.05 \%$ bovine serum albumin (Gibco; Thermo Fisher Scientific, Inc., Waltham, MA, USA), $0.05 \%$ Tween-20 and $0.0015 \%$ Triton X-100) and subsequently quenched with $3 \%$ peroxide-methanol at room temperature for $10 \mathrm{~min}$ for endogenous peroxidase ablation. Tissues were blocked in goat serum (1:100) at room temperature for $20 \mathrm{~min}$ prior to incubation with rabbit anti-human SUMO1 antibody (cat. no. ab32058; 1:1,000; Abcam, Cambridge, UK) for $2 \mathrm{~h}$ at $37^{\circ} \mathrm{C}$. Slides were rinsed in PBS-T (3x5 min) and biotin-labeled secondary antibody (cat. no. ab6720; Abcam), 1:500, was added for $30 \mathrm{~min}$ at $37^{\circ} \mathrm{C}$. After rinsing again in PBS-T $(3 \times 5 \mathrm{~min})$, slides were visualized by 3,3-diaminobenzidine tetrahydrochloride at room temperature without light for $10 \mathrm{~min}$ (Thermo Fisher Scientific, Inc.). Sections were counterstained using hematoxylin (Thermo Fisher Scientific, Inc.) for $1 \mathrm{~min}$ at room temperature. Sliders were dehydrated with sequential ethanol washes of 1 min each starting with $75 \%$, followed by $80 \%$ and finishing with a $100 \%$ ethanol wash. Images were captured using a BX51 microscope (Olympus Corporation, Tokyo, Japan).

Keratinocyte culture. The roofs of the blisters were washed twice with calcium-free Hanks' solution (Gibco; Thermo Fisher Scientific, Inc.), incubated with $0.25 \%$ trypsin solution (Gibco; Thermo Fisher Scientific, Inc.) at $37^{\circ} \mathrm{C}$ for $10 \mathrm{~min}$, followed by incubation with $0.02 \%$ EDTA solution (Gibco; Thermo Fisher Scientific, Inc.) at $37^{\circ} \mathrm{C}$ for $10 \mathrm{~min}$. Cells were separated from the epidermal sheet and observed under a stereo-microscope. The cell suspension was centrifuged $(1,000 \mathrm{x} g$ for $10 \mathrm{~min}$ at room temperature), resuspended with Medium 154 (Gibco; Thermo Fisher Scientific, Inc.) supplemented with Human Keratinocyte Growth Supplement (1:500; Gibco; Thermo Fisher Scientific, Inc.) with $100 \mathrm{U} / \mathrm{ml}$ penicillin and $0.1 \mathrm{mg} / \mathrm{ml}$ streptomycin. Cells were incubated in a $\mathrm{CO}_{2}$-regulated incubator in humidified $95 \%$ air $/ 5 \% \mathrm{CO}_{2}$ atmosphere at $37^{\circ} \mathrm{C}$ for approximately 3 weeks.

Short hairpin (sh)RNA sequences and transduction of HaCaT human keratinocytes. HaCat cells (American Type Culture Collection, Manassas, VA, USA) were cultured in DMEM supplemented with $10 \%$ fetal bovine serum (Gibco; Thermo Fisher Scientific, Inc.), $100 \mathrm{U} / \mathrm{ml}$ penicillin and $0.1 \mathrm{mg} / \mathrm{ml}$ streptomycin, in a $\mathrm{CO}_{2}$-regulated incubator in humidified $95 \%$ air $/ 5 \% \mathrm{CO}_{2}$ atmosphere at $37^{\circ} \mathrm{C}$. The lentiviral shRNA vectors included empty (cat. no. SHC001), non-targeting control (cat. no. SHC002) and SUMO1 (5'-CGACCAATGCAAGTGTTC ATA-3'; cat. no. PCA00244). The empty control consisted of any empty lentiviral vector without any shRNA, while the non-targeting control consisted of a lentiviral vector containing shRNA that does not target any known mammalian genes. Vectors were from the Sigma MISSON shRNA library (Sigma-Aldrich; Merck KGaA, Darmstadt, Germany). $\mathrm{HaCaT}$ cells were plated at a density of $5 \times 10^{6}$ in T75 flasks $20 \mathrm{~h}$ prior to infection. Each culture was infected at a MOI of 100 using HiPerFect transfection reagent (Qiagen $\mathrm{GmbH}$, Hilden, Germany). Transduced cells were selected with 
puromycin and subsequent experiments were performed $72 \mathrm{~h}$ after transduction.

Western blotting. Epidermis tissue samples, and patient-derived and HaCaT keratinocytes, were lysed in denaturing radioimmunoprecipitation assay (RIPA) lysis buffer (50 mM Tris, pH 8.0; 150 mM NaCl; 1\% NP-40; $1 \%$ sodium deoxycholate; $0.1 \%$ SDS) supplemented with 20 mM N-ethylmaleimide (Sigma-Aldrich; Merck KGaA) and protease and phosphatase inhibitors (Roche Diagnostics $\mathrm{GmbH}$, Mannheim, Germany). The protein concentration was determined with a bicinchoninic acid (BCA) protein assay. SDS-PAGE (10\%) was performed using the mini-gel system (Bio-Rad Laboratories, Inc., Hercules, CA, USA). Proteins were loaded at $20 \mu \mathrm{g} /$ lane and transferred onto nitrocellulose membranes. The membrane was blocked with $5 \%$ non-fat milk in TBS-Tween-20 (TBST) buffer (50 mM Tris-HCl, pH 7.6; $150 \mathrm{mM} \mathrm{NaCl} ; 0.1 \%$ Tween-20) for $1 \mathrm{~h}$ at room temperature, followed by overnight incubation at $4{ }^{\circ} \mathrm{C}$ with specific primary antibodies: SUMO1 (cat. no. 4930S; CST Biological Reagents Co., Ltd., Shanghai, China), SENP1 (cat. no. ab108981, Abcam), Ubc9 (cat. no. 4876S; CST Biological Reagents Co., Ltd.), SAE1 (cat. no. 13585S; CST Biological Reagents Co., Ltd.), cyclin-dependent kinase (CDK)2 (cat. no. ab32147; Abcam), CDK6 (cat. no. ab124821; Abcam), proliferating cell nuclear antigen (PCNA; cat. no. ab29; Abcam), retinoblastoma protein (Rb; cat. no. 9309S; CST Biological Reagents Co., Ltd.), phosphorylated Rb (pRb; cat. no. 9308S; CST Biological Reagents Co., Ltd.) and $\beta$-actin (cat. no. sc-47778; Santa Cruz Biotechnology, Inc., Dallas, TX, USA) at a 1:1,000 dilution. The membranes were washed with TBST buffer and incubated with IRDye $800 \mathrm{CW}$ goat anti-rabbit secondary antibody or IRDye 680 goat anti-mouse secondary antibody (LI-COR Biosciences, Lincoln, NE, USA) at a 1:10,000 dilution for $1 \mathrm{~h}$ at room temperature in the dark. The proteins were visualized using an Odyssey Infrared Imaging System (LI-COR Biosciences).

Detection of cell viability. Cell suspension (100 $\mu \mathrm{l} / \mathrm{well})$ of SUMO1 and non-targeting control shRNA-transduced HaCaT cells were seeded in 96-well plates at 5,000 cells/well. HaCaT cells were routinely cultured in the laboratory of the present study. This cell line is a spontaneously transformed aneuploid immortal keratinocyte cell line from adult human skin and is commonly used to investigate the function of keratinocytes. The plates were preincubated in an incubator at $37^{\circ} \mathrm{C}$ under $5 \% \mathrm{CO}_{2}$. Cell Counting Kit-8 (CCK-8) solution (10 $\mu \mathrm{l} /$ well; Dojindo Molecular Technologies, Inc., Kumamoto, Japan) was added to each well. The plate was incubated for $1-4 \mathrm{~h}$ at $37^{\circ} \mathrm{C}$. The absorbance at a wavelength of $450 \mathrm{~nm}$ was measured using a microplate reader.

Immunoprecipitation. Keratinocytes obtained from patients with vitiligo and healthy volunteers were lysed with RIPA buffer supplemented with protease and phosphatase inhibitors (Roche Diagnostics $\mathrm{GmbH}$ ) at $4^{\circ} \mathrm{C}$ for $30 \mathrm{~min}$. Protein concentration was determined using the $\mathrm{BCA}$ protein assay following centrifugation at $12,000 \mathrm{x} \mathrm{g}$ and $4^{\circ} \mathrm{C}$ for $10 \mathrm{~min} .1 \mathrm{mg}$ Proteins $(1 \mathrm{mg})$ were incubated with $2 \mu \mathrm{g}$ SUMO1 antibody (cat. no. 4930S; CST Biological Reagents Co., Ltd.) at 1:100 dilution for $16 \mathrm{~h}$ at $4^{\circ} \mathrm{C}$ with gentle inversion mixing. Subsequently, $50 \mu 1$ protein $\mathrm{A} / \mathrm{G}$ sepharose beads (Abmart, Inc., Berkeley Heights, NJ, USA) were added and incubated for $3 \mathrm{~h}$ at $4^{\circ} \mathrm{C}$. The beads were collected and washed four times with RIPA buffer. The immunoprecipitated proteins were eluted by $1 \mathrm{X}$ SDS sample buffer (Beyotime Institute of Biotechnology, Shanghai, China) and SUMO-1 conjugated Rb was detected by western blotting with Rb primary antibody (cat. no. 9309S; CST Biological Reagents Co., Ltd.) as described above.

Cell cycle examination. For cell cycle analysis, HaCaT keratinocytes were seeded at a density of $1 \times 10^{6}$ cell/well in 6 -well plates and fixed with prechilled $70 \%$ ethanol overnight at $4^{\circ} \mathrm{C}$ following transduction. Prior to analysis, cells were centrifuged $(1,000 \mathrm{x} \mathrm{g}$ for $10 \mathrm{~min}$ at room temperature) and resuspended in $1 \mathrm{ml}$ DNA staining solution (PBS containing $50 \mathrm{mg} / \mathrm{ml}$ propidium iodide and $200 \mathrm{mg} / \mathrm{ml} \mathrm{RNaseA)} \mathrm{and} 10 \mu \mathrm{l}$ permeabilization solution at room temperature for $30 \mathrm{~min}$ in the dark. Both DNA staining and permeabilization solution were included in the Cell Cycle Staining kit (Hangzhou MultiSciences Biotech Co., Ltd., Hangzhou, China). Cells were incubated at $37^{\circ} \mathrm{C}$ for $30 \mathrm{~min}$ and immediately assayed on a flow cytometer (FACS Calibur; BD Biosciences, Franklin Lakes, NJ, USA) with ModFit LT (version 4.1; Verity Software House, Inc., Topsham, ME, USA).

Statistical analysis. All data are presented as the mean \pm standard deviation of three independent experiments. Data were analyzed using unpaired Student's t-tests. SPSS version 17.0 (SPSS, Inc., Chicago, IL, USA) was used for all analyses. $\mathrm{P}<0.05$ was considered to indicate a statistically significant difference.

\section{Results}

SUMO1 conjugation is dysregulated in keratinocytes of patients with vitiligo. To analyze the SUMOylation in the keratinocytes of patients with vitiligo, the current study collected punch biopsies from vitiligo lesions of patients and skin specimens from healthy adults. SUMO1-conjugated proteins in epidermis tissues were investigated via IHC and western blotting. The results revealed decreased levels of SUMO1-conjugated proteins in the depigmented lesion tissues of patients with vitiligo compared with healthy control samples (Fig. 1A-C). These results indicate that SUMO1 conjugation may be dysregulated in the depigmented lesions of patients with vitiligo. To confirm this at the cellular level, primary keratinocytes were cultured from vitiligo lesions (KVs) and adult healthy skin (KNs) and western blot analysis was performed. The results demonstrated that SUMO1-conjugated proteins were markedly decreased in KVs compared with KNs (Fig. 1D). In addition, the protein expression of Ubc9, which is a SUMO-activating and -conjugating enzyme, was also markedly decreased in KVs compared with KNs, whereas no marked alteration in the expression of SAE1 was detected between the two groups. By contrast, the protein levels of SENP1, one of the SUMO-specific proteases involved in deSUMOylation, was increased in KVs compared with KNs (Fig. 1E). In summary, these results indicated that vitiligo lesions may exhibit dysregulated SUMOylation and deSUMOylation. 

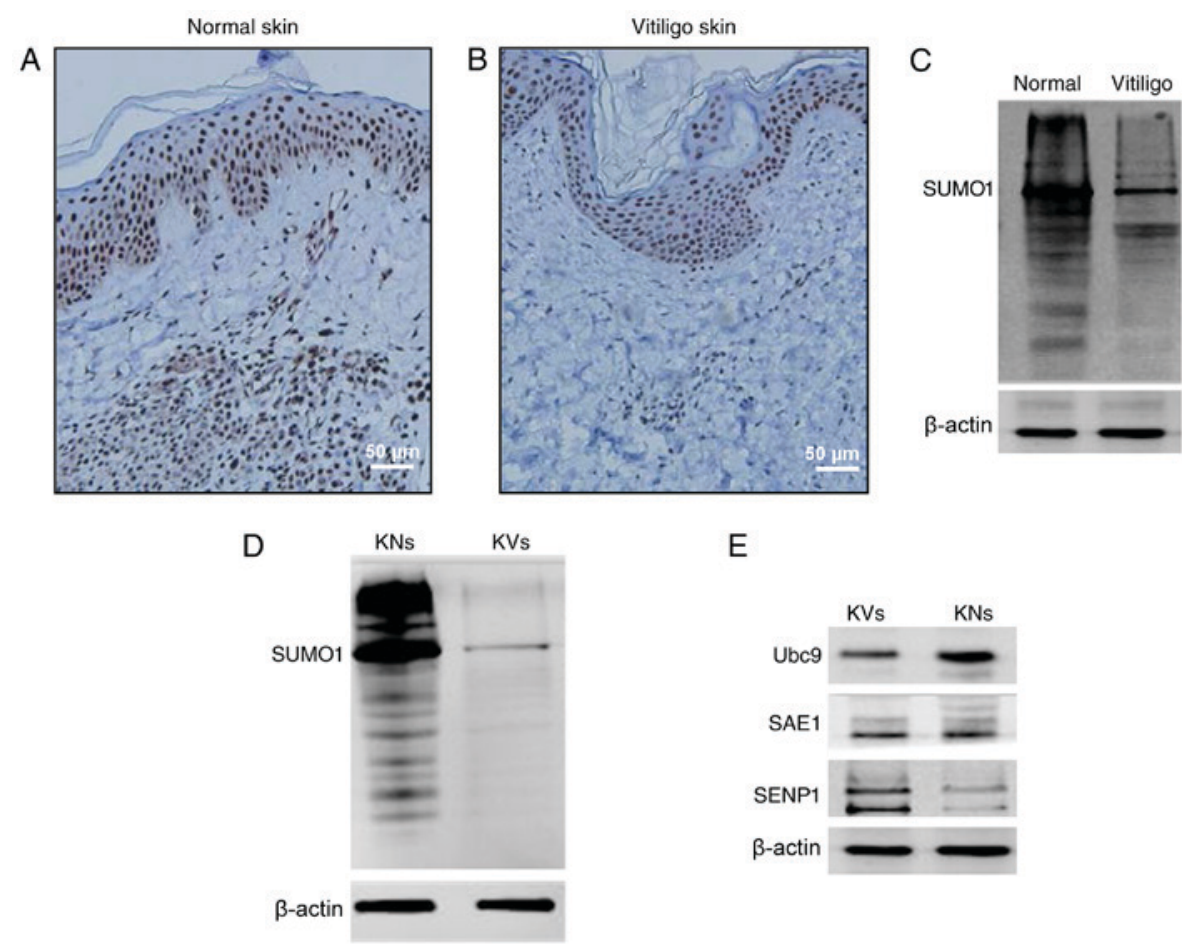

Figure 1. Dysregulation of SUMO1 conjugation in keratinocytes from vitiligo lesions. Immunohistochemical analysis of SUMO1-conjugated proteins in (A) healthy skin tissue and (B) vitiligo skin tissue. SUMO1-conjugated proteins were decreased in vitiligo skin tissue compared with levels in healthy skin tissue. Scale bar, $50 \mu \mathrm{m}$. (C) Western blot analysis of SUMO1-conjugated proteins in skin tissue obtained from healthy volunteers and patients with vitiligo. (D) Western blot analysis of SUMO1-conjugated proteins in primary keratinocytes cultured from healthy volunteers and patients with vitiligo. (E) Western blot analysis of SUMOylation-associated proteins in primary keratinocytes cultured from healthy volunteers and patients with vitiligo. The expression of Ubc9 was decreased and SENP1 was increased in vitiligo keratinocytes compared with healthy keratinocytes. SUMO1, small ubiquitin-like modifier 1; Ubc9, ubiquitin-conjugating enzyme E2 I; SENP1, SUMO-specific peptidase 1; KNs, healthy keratinocytes; KVs, vitiligo keratinocytes; SAE1, SUMO-activating enzyme subunit 1 .

Cell cycle is dysregulated in SUMOI knockdown HaCaT human keratinocytes. Western blot analysis demonstrated that following transduction of $\mathrm{HaCaT}$ cells with SUMO1 shRNA knockdown vectors, the expression of SUMO1 was partially knocked-down compared with non-transduced, empty vector and non-targeting shRNA control groups (Fig. 2A). The cell proliferation rate was determined by a CCK- 8 assay $72 \mathrm{~h}$ after transduction. The cell viability of SUMO1-knockdown $\mathrm{HaCaT}$ cells was demonstrated to be reduced compared with control cells transduced with non-targeting shRNA vectors at 48, 72 and 96 h (Fig. 2B). Furthermore, flow cytometry analysis demonstrated that the cell cycle was arrested in G1 phase in SUMO1-knockdown HaCaT cells compared with control $\mathrm{HaCaT}$ cells transduced with non-targeting shRNA (Fig. 2C and D). In addition, western blotting revealed decreased levels of CDK2, PCNA, CDK6 and pRb in SUMO1 knockdown $\mathrm{HaCaT}$ cells compared with control $\mathrm{HaCaT}$ cells transduced with non-targeting shRNA (Fig. 2E). PCNA is an important indicator of cell proliferation status. CDK2 and CDK6 belong to the CDK family, which drive the cell cycle through binding to their activating cyclins (17). $\mathrm{Rb}$ is a tumor suppressor, and its phosphorylation is essential for G0/G1 to $\mathrm{S}$ transition (18). These results indicate that dysregulation of cell cycle progression may be present in SUMO1 knockdown $\mathrm{HaCaT}$ human keratinocytes.

$R b$ SUMOylation is decreased in keratinocytes from lesions of vitiligo. To investigate the SUMOylation status of proteins associated with the cell cycle in keratinocytes from lesions of patients with vitiligo, cell lysates were subjected to immunoprecipitation with an anti-SUMO1 antibody and Rb antibody. It was demonstrated that SUMOylated $\mathrm{Rb}$ was markedly decreased in KVs compared with KNs, whereas the expression of Rb in cell lysates from both groups was equal (Fig. 3). These results indicate that the deSUMOylation of Rb in keratinocytes may serve an important role in vitiligo.

\section{Discussion}

To the best of our knowledge, the role of SUMOylation in the pathogenesis of vitiligo has not been reported previously. Therefore, the current study aimed to reveal abnormalities in SUMO conjugation in keratinocytes from depigmented lesions of patients with vitiligo and confirm the role of SUMOylation in the function of keratinocytes from patients with vitiligo. The results demonstrated that vitiligo lesions exhibited dysregulated SUMOylation and deSUMOylation. Furthermore, dysregulation of cell cycle progression was observed in SUMO1 knockdown HaCaT cells, and immunoprecipitation demonstrated that the SUMOylation of Rb in keratinocytes may be important in vitiligo.

Keratinocyte abnormalities have been reported to be implicated in the loss of adjacent melanocytes and the development of vitiligo, and keratinocyte apoptosis may result in the loss of survival stimuli and lead to passive melanocyte death $(2,5)$. SUMOylation, a form of post-translational modification, is 
A

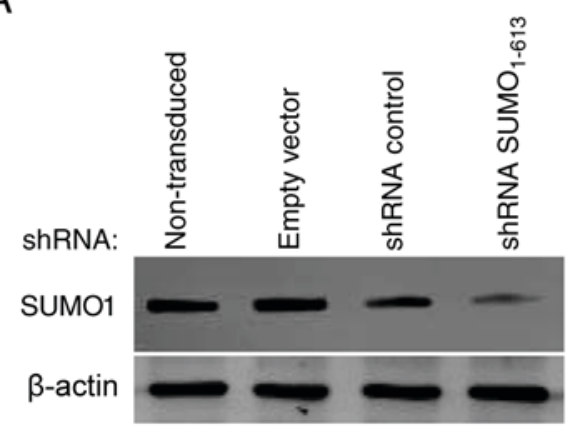

ShNA control

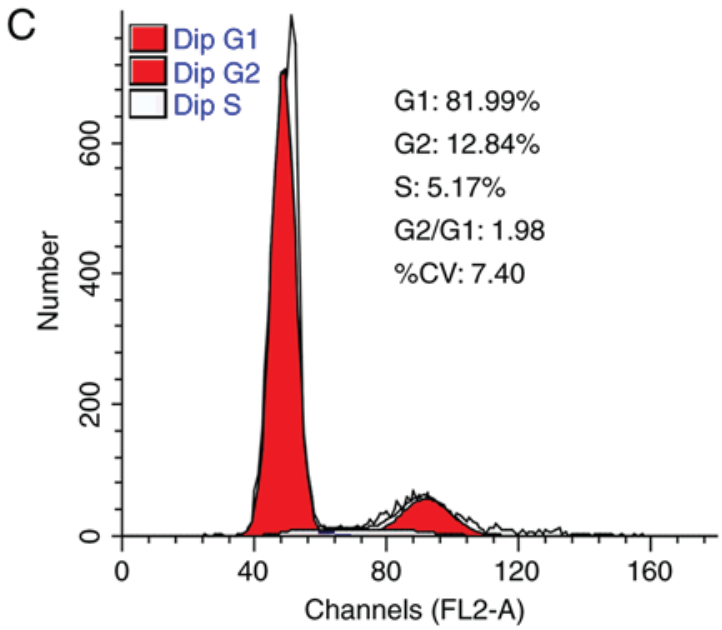

B

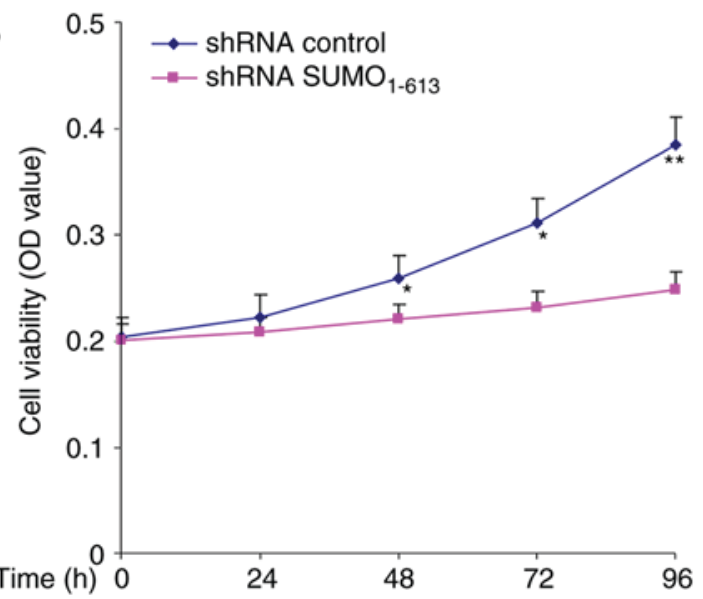

ShRNA SUMO $_{1-613}$

D

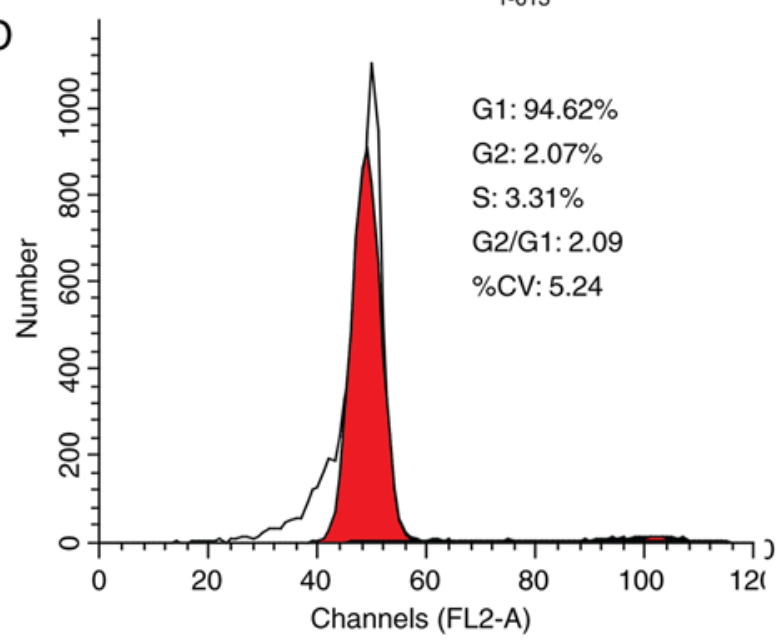

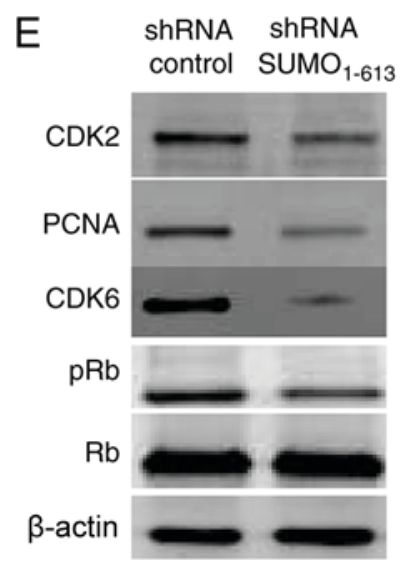

Figure 2. Proliferation rate and cell cycle analysis in SUMO1 knockdown HaCaT cells. (A) Western blotting confirmed that SUMO1 protein expression was successfully knocked-down in HaCaT cells transduced with SUMO1 shRNA lentiviral vectors. (B) A Cell Counting Kit-8 assay demonstrated that the proliferation rate of SUMO1-knockdown HaCaT cells was decreased compared with shRNA control-transduced cells. " $\mathrm{P}<0.05$, ${ }^{* *} \mathrm{P}<0.01$ vs. shRNA control group at same time-point. Propidium iodide staining and flow cytometry were performed for cell cycle analysis of (C) shRNA control-transduced and (D) SUMO1 knockdown HaCaT cells. The cell cycle of SUMO1 knockdown HaCaT cells was arrested in G1 phase. (E) Western blotting demonstrated that the expression of proteins associated with the cell cycle, including CDK2, PCNA, CDK6 and pRb, was decreased in SUMO1 knockdown HaCaT cells compared with shRNA control-transduced $\mathrm{HaCaT}$ cells. Total Rb expression was equal in both groups. shRNA control cells were transduced with non-targeting shRNA. SUMO1, small ubiquitin-like modifier 1; shRNA, short hairpin RNA; CDK, cyclin-dependent kinase; PCNA, proliferating cell nuclear antigen; Rb, retinoblastoma protein; $\mathrm{pRb}$, phosphorylated $\mathrm{Rb}$; OD, optical density.

essential for various biological processes, including roles in the DNA damage response, mitosis and cell cycle progression (2).
It was previously reported that knockout of Ubc9 or the SUMO E1 subunit SAE2 in U2OS and HCT116 human cancer cells 


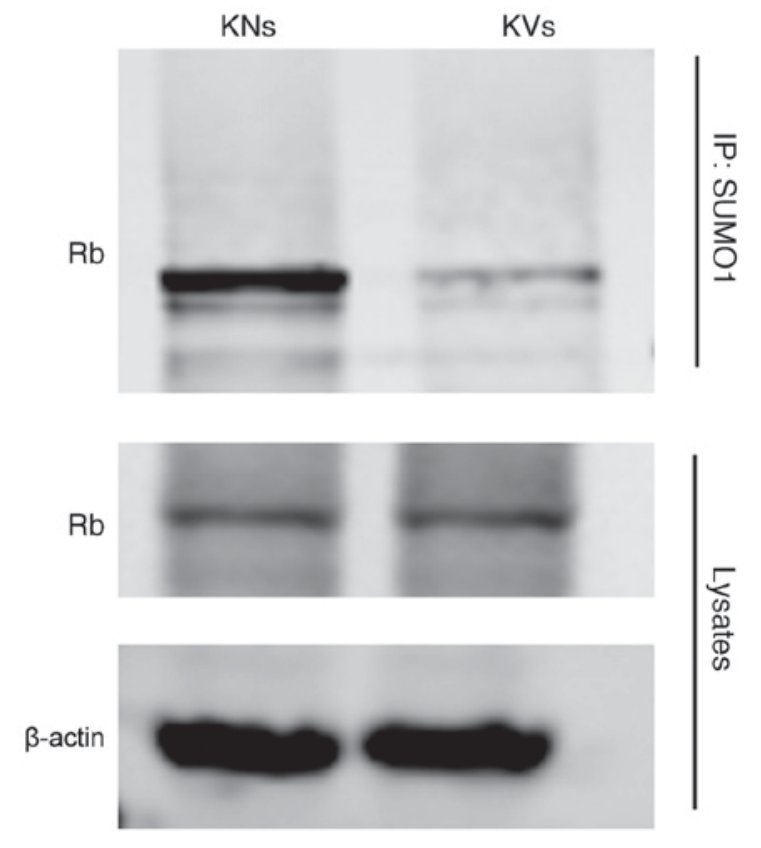

Figure 3. Immunoprecipitation analysis of SUMO1-conjugated Rb in primary keratinocytes cultured from healthy volunteers and patients with vitiligo. SUMO1-conjugated $\mathrm{Rb}$ was decreased in vitiligo keratinocytes compared with healthy keratinocytes. SUMO1, small ubiquitin-like modifier 1 ; Rb, retinoblastoma protein; KNs, healthy keratinocytes; KVs, vitiligo keratinocytes; IP, immunoprecipitate.

reduced the cell proliferation rate (19). In addition, the present study observed decreased viability in SUMO1 knockdown $\mathrm{HaCaT}$ cells. Furthermore, the cell cycle of $\mathrm{HaCaT}$ cells was arrested in G1 following SUMO1 knockdown. The G1 arrest of cell cycle following SUMO1 knockdown has also been observed in cancer cells (11).

CDKs are major components involved in cell cycle progression $(20,21)$. Proteomics studies have highlighted that CDKs and cyclins are targets of SUMO $(12,22)$. During mitosis, it has been reported that CDK6 SUMOylation may prevent the ubiquitination and subsequent degradation of CDK6, which means that CDK6 remains expressed during the G1/S transition (12). As CDKs are required for the progression of cells through the cell cycle (17), and SUMO1 knockdown was demonstrated to affect CDK2 and CDK6 expression in the present study, knockdown of SUMO1 may affect the cell cycle. SUMO1 is not essential for cell survival, as demonstrated by studies involving $\mathrm{SUMO}^{--}$mice, which indicated that SUMO2 may serve a compensatory role $(23,24)$. Additional studies are required to determine the contribution of the other SUMO family members to vitiligo.

$\mathrm{Rb}$ is a prototypical tumor suppressor that is involved in the negative regulation of the cell cycle and tumor progression. SUMO1-Rb has a role in G0/G1 to S phase transition and SUMOylation is reported to increase $\mathrm{Rb}$ phosphorylation (18). The phosphorylation of Rb during the G0/G1 to $\mathrm{S}$ transition has been demonstrated to be crucial in cell cycle regulation (25). In addition, PCNA has been reported to bind to SUMO1 (26). The present study reported markedly reduced SUMO1-conjugated $\mathrm{Rb}$ in keratinocytes from patients with vitiligo and the phosphorylation of $\mathrm{Rb}$ was also decreased in SUMO1 knockdown HaCaT cells. As pRb is essential for cell cycle progression, this effect of SUMO1 is likely to be involved in dysregulated keratinocytes.

The epidermis is composed of melanocytes and keratinocytes, and keratinocytes serve a vital role in the differentiation, proliferation and cell viability of melanocytes, which indicates that keratinocytes are important in the homeostasis of the epidermis via direct cell-to-cell contact and the secretion of growth factors (2). The results of the present study indicated that dysregulated SUMO1 in keratinocytes may lead to a dysregulated cell cycle and may affect other cell functions. Therefore, these abnormal keratinocytes may be unable to support the survival of melanocytes. However, the mechanism by which SUMO1 dysregulation leads to impaired functioning of keratinocytes, and how SUMO1-dysregulated keratinocytes affect the survival of melanocytes, requires further investigation.

The present study is not without limitations. Only a small panel of proteins was assessed. In addition, no mRNA analysis was performed. Future studies should also investigate how SUMO1 knockdown keratinocytes interact with melanocytes in co-culture experiments or animal models. Additional studies are required to understand the underlying mechanisms that lead to vitiligo.

In conclusion, in the present study, vitiligo lesions exhibited dysregulated SUMOylation and deSUMOylation in keratinocytes, dysregulation of the cell cycle progression was observed in SUMO1 knockdown HaCaT cells and the deSUMOylation of $\mathrm{Rb}$ in keratinocytes may serve an important role in the development of vitiligo. The results of the present study provide a future direction for research into the mechanism of vitiligo, as well as novel targets for therapy development.

\section{Acknowledgements}

The authors acknowledge the assistance of Professor Honglin Wang from Shanghai Jiaotong University School of Medicine (Shanghai, China) for assistance with the immunohistochemistry and immunoprecipitation experiments.

\section{Funding}

The current study was supported by grants from the National Natural Science Foundation of China (grant nos. 81472887, 81773335 and 81602755), Zhejiang Provincial Natural Science Foundation (grant nos. LY18H110001 and LQ16H290001) and Zhejiang Basic Public Welfare Research Project (grant no. LGF18H110002).

\section{Availability of data and materials}

The datasets used and/or analyzed during the current study are available from the corresponding author on reasonable request.

\section{Authors' contributions}

MZ analyzed SUMOylation levels in the vitiligo lesions, SUMO-1 knockdown in $\mathrm{HaCaT}$ cells and was a major contributor in writing the manuscript. FL performed shRNA knockdown of SUMO-1 and the immunoprecipitation experiments. WX performed IHC, western blot and cell cycle 
analysis. RJ cultured the cells and detected cell viability. AX provided the patient samples and interpreted the data. All authors read and approved the final manuscript.

\section{Ethics approval and consent to participate}

The present study was approved by the Institutional Review Board of the Third People's Hospital of Hangzhou (Hangzhou, China). Informed consent was obtained from each participant.

\section{Patient consent for publication}

Not applicable.

\section{Competing interests}

The authors declare that they have no competing interests.

\section{References}

1. Lazzeri L, Colucci R, Cammi A, Dragoni F and Moretti S: Adult onset vitiligo: Multivariate analysis suggests the need for a thyroid screening. Biomed Res Int 2016: 8065765, 2016

2. Lee AY: Role of keratinocytes in the development of vitiligo. Ann Dermatol 24: 115-125, 2012.

3. Hirobe T: Keratinocytes regulate the function of melanocytes. Dermatologica Sinica 32: 200-204, 2014.

4. Shin YH, Seo YK, Yoon HH, Song KY and Park JK: Effect of keratinocytes on regulation of melanogenesis in culture of melanocytes. Biotech Bioproc Eng 17: 203-210, 2012.

5. Prignano F, Pescitelli L, Becatti M, Di Gennaro P, Fiorillo C, Taddei $\mathrm{N}$ and Lotti T: Ultrastructural and functional alterations of mitochondria in perilesional vitiligo skin. J Dermatol Sci 54 157-167, 2009.

6. Lee AY, Kim NH, Choi WI and Youm YH: Less keratinocyte-derived factors related to more keratinocyte apoptosis in depigmented than normally pigmented suction-blistered epidermis may cause passive melanocyte death in vitiligo. J Invest Dermatol 124: 976-983, 2005.

7. Raman N, Nayak A and Muller S: The SUMO system: A master organizer of nuclear protein assemblies. Chromosoma 122 $475-485,2013$

8. Schimmel J, Eifler K, Sigurðsson JO, Cuijpers SA, Hendriks IA, Verlaan-de Vries M, Kelstrup CD, Francavilla C, Medema RH, Olsen JV and Vertegaal AC: Uncovering SUMOylation dynamics during cell-cycle progression reveals FoxM1 as a key mitotic SUMO target protein. Mol Cell 53: 1053-1066, 2014.

9. Cubeñas-Potts C and Matunis MJ: SUMO: A multifaceted modifier of chromatin structure and function. Dev Cell 24: 1-12, 2013.
10. Chen $\mathrm{CH}$, Chang CC, Lee TH, Luo M, Huang P, Liao PH, Wei S, Li FA, Chen RH, Zhou XZ, et al: SENP1 deSUMOylates and regulates Pin1 protein activity and cellular function. Cancer Res 73: 3951-3962, 2013.

11. He X, Riceberg J, Pulukuri SM, Grossman S, Shinde V, Shah P, Brownell JE, Dick L, Newcomb J and Bence N: Characterization of the loss of SUMO pathway function on cancer cells and tumor proliferation. PLoS One 10: e0123882, 2015.

12. Eifler K and Vertegaal ACO: SUMOylation-mediated regulation of cell cycle progression and cancer. Trends Biochem Sci 40 779-793, 2015.

13. Bellail AC, Olson JJ and Hao C: SUMO1 modification stabilizes CDK6 protein and drives the cell cycle and glioblastoma progression. Nat Commun 5: 4234, 2014.

14. Deyrieux AF, Rosas-Acosta G, Ozbun MA and Wilson VG: Sumoylation dynamics during keratinocyte differentiation. J Cell Sci 120: 125-136, 2007.

15. Taieb A, Alomar A, Böhm M, Dell'anna ML, De Pase A, Eleftheriadou V, Ezzedine K, Gauthier Y, Gawkrodger DJ, Jouary T, et al: Guidelines for the management of vitiligo: The European dermatology forum consensus. Br J Dermatol 168: 5-19, 2013.

16. Taïeb A and Picardo M: Clinical practice. Vitiligo. N Engl J Med 360: 160-169, 2009.

17. Lim S and Kaldis P: Cdks, cyclins and CKIs: Roles beyond cell cycle regulation. Development 140: 3079-3093, 2013.

18. Meng F, Qian J, Yue H, Li X and Xue K: SUMOylation of Rb enhances its binding with CDK2 and phosphorylation at early G1 phase. Cell Cycle 15: 1724-1732, 2016.

19. Kim KI and Baek SH: Small ubiquitin-like modifiers in cellular malignancy and metastasis. Int Rev Cell Mol Biol 273: 265-311, 2009.

20. Neyret-Kahn H, Benhamed M, Ye T, Le Gras S, Cossec JC, Lapaquette P, Bischof O, Ouspenskaia M, Dasso M, Seeler J, et al: Sumoylation at chromatin governs coordinated repression of a transcriptional program essential for cell growth and proliferation. Genome Res 23: 1563-1579, 2013.

21. Asghar U, Witkiewicz AK, Turner NC and Knudsen ES: The history and future of targeting cyclin-dependent kinases in cancer therapy. Nat Rev Drug Discov 14: 130-146, 2015.

22. Hendriks IA, D'Souza RC, Yang B, Verlaan-de Vries M, Mann M and Vertegaal AC: Uncovering global SUMOylation signaling networks in a site-specific manner. Nat Struct Mol Biol 21: 927-936, 2014

23. Evdokimov E, Sharma P,Lockett SJ,Lualdi M and Kuehn MR: Loss of SUMO1 in mice affects RanGAP1 localization and formation of PML nuclear bodies, but is not lethal as it can be compensated by SUMO2 or SUMO3. J Cell Sci 121: 4106-4113, 2008.

24. Zhang FP, Mikkonen L, Toppari J, Palvimo JJ, Thesleff I and Jänne OA: Sumo-1 function is dispensable in normal mouse development. Mol Cell Biol 28: 5381-5390, 2008.

25. Giacinti C and Giordano A: RB and cell cycle progression. Oncogene 25: 5220-5227, 2006.

26. Armstrong AA, Mohideen F and Lima CD: Recognition of SUMO-modified PCNA requires tandem receptor motifs in Srs2. Nature 483: 59-63, 2012. 